\title{
Dengue Virus and Yellow Fever Virus Detection Using Reverse Transcription-Insulated Isothermal PCR and Comparison with Real-Time RT-PCR
}

\author{
Victoria Stittleburg, ${ }^{1}$ Alejandra Rojas, ${ }^{2}$ Fátima Cardozo, ${ }^{3}$ Flor M. Muñoz, ${ }^{4}$ Edwin J. Asturias, ${ }^{5,6}$ Daniel Olson, ${ }^{5}$ \\ Alejandra Paniaga-Avila, ${ }^{6}$ Janaki Abeynayake, ${ }^{7}$ Evan J. Anderson, ${ }^{1,8}$ and Jesse J. Waggoner ${ }^{1,9 *}$ \\ ${ }^{1}$ Division of Infectious Diseases, Department of Medicine, Emory University, Atlanta, Georgia; ${ }^{2}$ Departamento de Producción, Instituto de \\ Investigaciones en Ciencias de la Salud, Universidad Nacional de Asunción, San Lorenzo, Paraguay; ${ }^{3}$ Departamento de Salud Pública, Instituto de \\ Investigaciones en Ciencias de la Salud, Universidad Nacional de Asunción, San Lorenzo, Paraguay; ${ }^{4}$ Departments of Pediatrics and Molecular \\ Virology and Microbiology, Baylor College of Medicine, Houston, Texas; ${ }^{5}$ Division of Infectious Diseases and Epidemiology, Department of \\ Pediatrics, University of Colorado at Denver, Aurora, Colorado; ${ }^{6}$ Fundación para la Salud Integral de los Guatemaltecos, FUNSALUD, \\ Quetzaltenango, Guatemala; ${ }^{7}$ Medical Research Institute, Colombo, Sri Lanka; ${ }^{8}$ Division of Infectious Diseases, Department of Pediatrics, Emory \\ University, Atlanta, Georgia; ${ }^{9}$ Department of Global Health, Rollins School of Public Health, Atlanta, Georgia
}

\begin{abstract}
Real-time reverse transcriptase PCR (rRT-PCR) is the most accurate method for the detection of dengue virus (DENV) and yellow fever virus (YFV) in acute illness. However, performing rRT-PCR is not feasible for many laboratories in regions of endemicity. The current study compared new reverse transcription-insulated isothermal PCRs (the POCKIT DENV and YFV reagent sets) with laboratory-developed rRT-PCRs for both viruses using clinical samples and viral strains from different endemic regions. Sensitivity and specificity of the POCKIT DENV Reagent Set were 87.2\% (68/ 78 samples) and $98.2 \%$ of samples (54/55), respectively. The YFV reagent set demonstrated sensitive detection of YFV RNA from six viral strains down to an estimated concentration of $2.5 \log _{10}$ copies/mL and proved to be specific for YFV. Although the POCKIT assays require RNA extraction, they may provide accurate and less-complex options for molecular testing in laboratory settings where rRT-PCR is not practical.
\end{abstract}

The genus Flavivirus includes dengue virus (DENV), the most common human arbovirus worldwide, and yellow fever virus (YFV), which remains a significant public health threat despite the availability of an effective vaccine. ${ }^{1-4}$ Both pathogens present diagnostic challenges in regions of endemicity. ${ }^{3-6}$ Real-time reverse transcriptase PCR (rRTPCR) is the most accurate method for DENV and YFV detection during acute infection, although viral RNA may only be detectable in serum or plasma for 5-7 days after symptom onset. ${ }^{4,6}$ In resource-limited settings, few laboratories have the capacity to perform high-complexity molecular testing, and results from national or regional reference laboratories are not available in a clinically relevant time frame. As a result, patients may receive a clinical diagnosis that is inaccurate for differentiating DENV or YFV from many other causes of acute febrile illness. ${ }^{2-4}$ Timely diagnosis and initiation of management for dengue improve clinical outcomes. ${ }^{2}$ Similar data are not available for yellow fever, where early detection may be particularly important, given the high case-fatality rate for severe cases. ${ }^{4}$ Rapid diagnostics are available for the detection of DENV nonstructural protein 1 (NS1) and anti-DENV IgM, but these are significantly less sensitive than rRT-PCR during acute infection. 6,7 Testing for anti-YFV IgM is often limited to reference laboratories. For both DENV and YFV, IgM is not reliably detectable until 5 days after symptom onset, and significant cross-reactivity occurs between flaviviruses. , $^{3,6,8}$

Recently, a hydrolysis probe-based reverse transcriptioninsulated isothermal PCR has been developed for the detection of DENV in serum (POCKIT DENV Reagent Set, GeneReach Biotechnology, Taichung City, Taiwan). ${ }^{9-13}$ This assay can be performed on a handheld battery-powered instrument that is inexpensive relative to the costs of real-time PCR instruments. Although assay comparisons between the

*Address correspondence to Jesse J. Waggoner, Division of Infectious Disease, Emory University, 1760 Haygood Dr. NE, Rm. E-169, Atlanta, GA 30322. E-mail: jjwaggo@emory.edu
DENV reagent set and an FDA-cleared DENV rRT-PCR have been reported, ${ }^{9,10}$ independent evaluations of this assay have not been published. In addition, no data have been published regarding the performance of the POCKIT YFV Reagent Set. The objectives of the current study were to evaluate both reagent sets using a diverse set of clinical samples and/or reference viral strains from different regions of endemicity and to compare assay performance with sensitive laboratorydeveloped rRT-PCRs for each pathogen. ${ }^{14,15}$

Evaluation of the POCKIT DENV Reagent Set was performed using pre-collected clinical samples from sites in Guatemala (DENV-2 and -3), Paraguay (DENV-1 and -4), and Sri Lanka (DENV-2). Samples had been obtained from patients with a suspected arboviral illness within the first 7 days of symptom-onset as part of ongoing arboviral studies (Guatemala and Paraguay) or routine clinical care (Sri Lanka). Sera were collected in Paraguay and Sri Lanka, whereas plasma and whole blood samples were collected in Guatemala. This research protocol was reviewed and approved by the Emory University Institutional Review Board (Protocol 91162). All statistical analyses were performed using GraphPad software (v. 8.1.1, GraphPad, San Diego, CA).

Total nucleic acids were extracted on an eMAG instrument (bioMérieux, Marcy-l'Étoile, France) according to the manufacturer-recommended protocols. Samples were initially screened using an rRT-PCR for Zika virus, chikungunya virus, and DENV (the "ZCD assay"). ${ }^{16}$ Dengue virus-positive samples were then confirmed in a quantitative, serotypespecific DENV rRT-PCR. ${ }^{15}$ Nucleic acids were stored at $-80^{\circ} \mathrm{C}$ until tested once with the POCKIT DENV Reagent Set on a Micro Plus Nucleic Acid Analyzer according to the manufacturer-supplied protocol. ${ }^{9}$ Samples with discordant rRT-PCR and POCKIT results were tested a second time with the serotype-specific DENV rRT-PCR for confirmation.

One hundred thirty-three samples were tested for DENV (serum/plasma, $n=118$; whole blood, $n=15$ ). Overall results are shown in Table 1. Four samples (3.0\%) yielded "undetermined" results, which display as a "?" on the nucleic acid 
TABLE 1

Comparison of the POCKIT DENV Reagent Set with a laboratorydeveloped rRT-PCR

\begin{tabular}{|c|c|c|c|c|}
\hline \multirow{2}{*}{$\begin{array}{c}\text { DENV rRT-PCR } \\
\text { Result }\end{array}$} & \multirow[b]{2}{*}{$N$} & \multirow{2}{*}{$\frac{\text { Viral load }}{\text { Median (range) }^{\star}}$} & \multicolumn{2}{|c|}{ Results, POCKIT DENV } \\
\hline & & & Detected, $n$ & Sensitivity (\%) \\
\hline DENV positive & 78 & $5.2(2.7-10.8)$ & 68 & 87.2 \\
\hline DENV-1 & 24 & $5.3(2.7-9.3)$ & 20 & 83.3 \\
\hline DENV-2 & 33 & $4.4(2.8-10.8)$ & 29 & 87.9 \\
\hline DENV-3 & 18 & $6.8(4.2-9.5)$ & $16 \dagger$ & 88.9 \\
\hline DENV-4 & 3 & $4.8(4.8-5.5)$ & 3 & 100 \\
\hline DENV negative & 55 & - & $1 \ddagger$ & - \\
\hline
\end{tabular}

analyzer. Undetermined samples included 3 DENV-positive samples (DENV-1 and DENV-2, $n=2$ ) and 1 DENV-negative sample. These were retested with the POCKIT assay: 3/4 yielded negative/undetermined results and one sample yielded one positive and one negative result. All undetermined samples were thus classified as negative for further calculations.

The POCKIT assay demonstrated a high level of agreement with reference $\mathrm{rRT}-\mathrm{PCR}$ results (kappa statistic $0.83,95 \% \mathrm{Cl}$ $0.74-0.93)$, and the sensitivity was $87.2 \%$ (68/78). Seven samples were negative in the POCKIT assay but positive by rRT-PCR. When combined with undetermined samples, 8/10 samples that tested positive by rRT-PCR but negative/ undetermined in the POCKIT had viral loads less than 4.0 $\log _{10}$ copies/mL serum (DENV-1, $n=4$; DENV-2, $n=4$ ). Two DENV-3 samples gave false-negative results and included one plasma and one whole blood sample with viral loads of 4.2 and $5.4 \log _{10}$ copies/mL, respectively. Therefore, the POCKIT assay detected $96.2 \%$ of samples with viral loads $\geq 4.0 \log _{10}$ copies/mL (50/52). Specificity of the POCKIT assay was $98.2 \%(54 / 55)$, which resulted from one positive serum sample that was repeatedly negative in both the ZCD and DENV serotype-specific rRT-PCRs.

Evaluation of the POCKIT YFV Reagent Set was performed using dilutions of genomic RNA from six reference YFV strains representing five genotypes (Table 2). Dilutions were tested in duplicate across a range of concentrations extending from 1.0 to $9.3 \log _{10}$ copies/mL of sample. Viral load was quantified in a laboratory-developed rRT-PCR. ${ }^{14}$ For dilutions with viral loads $\geq 2.5 \log _{10}$ copies $/ \mathrm{mL}(\sim 1$ copy $/ \mu \mathrm{L}$ of eluate), RNA detection in the POCKIT YFV Reagent Set (43/48, 89.6\%) was similar to that in the laboratory-developed rRT-PCR $(44 / 48$, $91.7 \%)$. Of samples with viral loads below this level, 1/12 $(8.3 \%)$ was detected in the POCKIT assay compared with 5/12 $(41.7 \%)$ by rRT-PCR. To evaluate assay specificity, genomic RNA was tested from related flaviviruses (Table 2$).{ }^{14}$ In addition, 12 DENV-positive and 12 DENV-negative serum samples from Paraguay were tested. All samples in the specificity analysis tested negative except for one DENV-1 sample that yielded an indeterminate result. This sample tested negative for YFV by rRT-PCR and was negative in the POCKIT YFV Reagent Set on repeat testing. ${ }^{14}$

In this study, the POCKIT DENV and YFV reagent sets were compared with laboratory-developed rRT-PCRs that have proven to be more sensitive than common comparators for each pathogen. ${ }^{14,15,17}$ The POCKIT DENV assay demonstrated a sensitivity of $87.2 \%$ and detected $96.2 \%$ of DENVpositive samples with viral loads $\geq 4.0 \log _{10}$ copies $/ \mathrm{mL}$. Viral loads in this range are common among patients who present within the first 5 days of symptom onset, ${ }^{7}$ such that assay use could be targeted to a high-yield population based on information that is readily available at presentation. Notably, sensitivity is higher than that of a rapid NS1 assay used to test patients in Paraguay (71.4\%). ${ }^{7}$ Given the rapidity of assay performance (setup time, 5-10 minutes; run time, 45 minutes), this could provide a sensitive testing option for use near the point of patient care.

Assay performance of the YFV reagent set has not yet been published, but in this initial evaluation, the assay proved to be as sensitive as rRT-PCR down to viral loads of $2.5 \log _{10}$ copies/mL using genomic RNA from six YFV strains (five genotypes). A single DENV-1 sample gave an indeterminate result in the YFV assay, although this was negative on repeat testing. Given the high significance of detecting even a single yellow fever case, which can trigger vaccination campaigns and the issuance of travel medicine advisories, reference YFV testing with rRT-PCR testing will need to be available to confirm results. ${ }^{3,4,8}$ However, this test may provide an option for sensitive molecular testing in remote and/or resourcelimited regions disproportionately affected by YFV., ${ }^{3,4}$ As such, it warrants further evaluation with acute-phase clinical samples. ${ }^{4}$

Currently, the manufacturer only recommends the use of serum with these two reagent sets, although one previous

TABLE 2

Evaluation of POCKIT YFV reagent set

\begin{tabular}{|c|c|c|c|}
\hline \multirow[b]{2}{*}{ Sample } & \multirow[b]{2}{*}{ Viral load, range ${ }^{\star}$} & \multirow{2}{*}{$\frac{\text { YFV real-time reverse transcriptase PCR }}{\text { Detected, } n / \text { total }(\%)}$} & \multirow{2}{*}{$\frac{\text { POCKIT yellow fever }}{\text { Detected, } n / \text { total (\%) }}$} \\
\hline & & & \\
\hline Sensitivity evaluation & $\geq 5.5$ & $22 / 22(100)$ & $22 / 22(100)$ \\
\hline & $3.25-5.49$ & $14 / 14(100)$ & $14 / 14(100)$ \\
\hline YFV strainst & $2.5-3.24$ & $8 / 12(66.7)$ & $7 / 12(58.3)$ \\
\hline & $1.0-2.49$ & $5 / 12(41.7)$ & $1 / 12(8.3)$ \\
\hline \multicolumn{4}{|l|}{ Specificity evaluation } \\
\hline Flaviviral strainsł & $7.2-8.8$ & $0 / 8$ & $0 / 8$ \\
\hline DENV-positive samples§ & $5.1-9.3$ & $0 / 12$ & $0 / 12$ \\
\hline DENV-negative samples & - & $0 / 12$ & $0 / 12$ \\
\hline $\begin{array}{l}\text { DENV = dengue virus; YFV = yellow } \\
\text { * Expressed as log }{ }_{10} \text { copies/mL of } \\
\text { † Yellow fever virus strains: Asibi-G } \\
\text { 10a-10- Venezuela } 2010 \text { (South Ame } \\
\text { ‡Genomic RNA from West Nile viru } \\
\text { §DENV-1 ( } n=11 \text { ) and DENV-4 ( } n= \\
\text { serum). }\end{array}$ & $\begin{array}{l}\text { rD } 149194,1996 \\
\text { livia } 2006 \text { (South } \\
\text { encephalitis viru } \\
\text { le yielded an initi }\end{array}$ & $\begin{array}{l}\text { Couma-Ethiopia } 1961 \text { (East/Central Afric } \\
\text { irus, St. Louis encephalitis virus (two str } \\
\text { FV result that was negative on repeat te }\end{array}$ & $\begin{array}{l}2016 \text { (Angola); INHRR } \\
\text { NV-4. } \\
8.1 \log _{10} \text { copies } / \mathrm{mL} \text { of }\end{array}$ \\
\hline
\end{tabular}


publication also used plasma. ${ }^{9}$ It is unclear if this difference in the sample matrix affects performance, but notably, the DENV-3 samples that tested negative in the POCKIT assay were plasma and whole blood. The POCKIT assay still requires nucleic acid extraction, which in turn requires the availability of skilled personnel and relatively sophisticated laboratory facilities that can maintain molecular workflow. However, additional instruments are available for automated RNA extraction and sample-to-answer detection based on this technology. ${ }^{11,12}$ In conclusion, the POCKIT DENV and YFV reagent sets compared favorably with sensitive $\mathrm{RRT}$-PCRs for each pathogen, and these may provide accurate and lesscomplex options for molecular testing in certain laboratory settings.

Received December 3, 2019. Accepted for publication April 22, 2020.

Published online May 26, 2020.

Acknowledgments: We thank Yvalena de Guillén, Laura Mendoza, Cynthia Bernal, César Cantero, and all members of the study team based at the Instituto de Investigaciones en Ciencias de la Salud, Asunción, Paraguay. We also thank the study team at the Fundación para la Salud Integral de los Guatemaltecos, FUNSALUD, Quetzaltenango, Guatemala. We are grateful to all study participants and their families. Samples from Guatemala were used by permission of the National Institute of Allergy and Infectious Diseases (NIAID), National Institutes of Health (NIH). We thank Walla Dempsey and Kay Tomashek for their assistance in this process.

Financial Support: This project has been funded in whole or in part with Federal funds from the National Institute of Allergy and Infectious Diseases (NIAID). Research was supported by National Institutes of Health $(\mathrm{NIH})$, Department of Health and Human Services (under grant K08Al110528 [JJW]), and a NIAID DMID Vaccine and Treatment Evaluation Unit (VTEU) award to Baylor College of Medicine (Contract No. HHSN27220130015I).

Disclosure: E. J. Anderson has received personal fees from AbbVie and Pfizer for consulting, and his institution received funds to conduct clinical research unrelated to this study from Medlmmune, Regeneron, PaxVax, Pfizer, GSK, Merck, Novavax, Sanofi-Pasteur, and Micron.

Authors' addresses: Victoria Stittleburg, Department of Medicine, Emory University, Atlanta, GA, E-mail: victoria.d.simmons@emory. edu. Alejandra Rojas, Departamento de Producción, Instituto de Investigaciones en Ciencias de la Salud, Universidad Nacional de Asunción, San Lorenzo, Paraguay, E-mail: arojass@iics.una.py. Fátima Cardozo, Departamento de Salud Pública, Instituto de Investigaciones en Ciencias de la Salud, Universidad Nacional de Asunción, San Lorenzo, Paraguay, E-mail: fati.cardozo@hotmail.com. Flor M. Muñoz, Departments of Pediatrics and Molecular Virology and Microbiology, Baylor College of Medicine, Houston, TX, E-mail: florm@bcm.edu. Edwin J. Asturias, Department of Pediatrics, University of Colorado, Aurora, CO, and Center for Global Health, University of Colorado, Aurora, CO, E-mail: edwin.asturias@children scolorado.org. Daniel Olson, Division of Infectious Diseases and Epidemiology, Department of Pediatrics, University of Colorado, Aurora, $\mathrm{CO}$, and Colorado School of Public Health, Center for Global Health, E-mail: daniel.olson@childrenscolorado.org. Alejandra Paniaga-Avila, Center for Human Development, Fundación para la Salud Integral de los Guatemaltecos, FUNSALUD, Quetzaltenango, Guatemala, E-mail: alejandra.paniagua.fsigcu@gmail.com. Janaki Abeynayake, Medical Research Institute, Colombo, Sri Lanka, E-mail: janakiiabeynayake@ yahoo.com. Evan J. Anderson, Department of Medicine, Emory University, Atlanta, GA, E-mail: evanderson@emory.edu. Jesse J. Waggoner, Division of Infectious Diseases, Emory University, Atlanta, GA, E-mail: jjwaggo@emory.edu.

\section{REFERENCES}

1. Kuno G, Chang GJ, Tsuchiya KR, Karabatsos N, Cropp CB, 1998. Phylogeny of the genus Flavivirus. J Virol 72: 73-83.

2. World Health Organization, 2009. Dengue: Guidelines for Diagnosis, Treatment, Prevention and Control. Geneva, Switzerland: WHO Press.

3. Monath TP, Vasconcelos PF, 2015. Yellow fever. J Clin Virol 64: 160-173.

4. Waggoner JJ, Rojas A, Pinsky BA, 2018. Yellow fever virus: diagnostics for a persistent arboviral threat. J Clin Microbiol 56: e00827-18.

5. Wang WK, Gubler DJ, 2018. Potential point-of-care testing for dengue virus in the field. J Clin Microbiol 56: e00203-18.

6. Peeling RW et al., 2010. Evaluation of diagnostic tests: dengue. Nat Rev Microbiol 8 (12 Suppl): S30-S38.

7. Rojas $A$ et al., 2019. Characterization of dengue cases among patients with an acute illness, Central Department, Paraguay. PeerJ 7: e7852.

8. Pan American Health Organization, 2018. Laboratory Diagnosis of Yellow Fever Virus Infection. Washington, D.C.

9. Go $Y Y$ et al., 2016. A pan-dengue virus reverse transcriptioninsulated isothermal PCR assay intended for point-of-need diagnosis of dengue virus infection by use of the POCKIT nucleic acid analyzer. J Clin Microbiol 54: 1528-1535.

10. Tsai JJ, Liu LT, Lin PC, Tsai CY, Chou PH, Tsai YL, Chang HG, Lee $P A, 2018$. Validation of the pockit dengue virus reagent set for rapid detection of dengue virus in human serum on a fielddeployable PCR system. J Clin Microbiol 56: e01865-17.

11. Tsai JJ et al., 2019. An RT-PCR panel for rapid serotyping of dengue virus serotypes 1 to 4 in human serum and mosquito on a field-deployable PCR system. PLoS One 14: e0214328.

12. Tsai JJ et al., 2019. A fully automated sample-to-answer PCR system for easy and sensitive detection of dengue virus in human serum and mosquitos. PLoS One 14: e0218139.

13. Tsai YL, Wang HT, Chang HF, Tsai CF, Lin CK, Teng PH, Su C, Jeng CC, Lee PY, 2012. Development of TaqMan probe-based insulated isothermal PCR (iiPCR) for sensitive and specific onsite pathogen detection. PLoS One 7: e45278.

14. Rojas A et al., 2018. Internally controlled, multiplex real-time reverse transcription PCR for dengue virus and yellow fever virus detection. Am J Trop Med Hyg 98: 1833-1836.

15. Waggoner JJ et al., 2013. Single-reaction, multiplex, real-time rtPCR for the detection, quantitation, and serotyping of dengue viruses. PLoS Negl Trop Dis 7: e2116.

16. Waggoner JJ, Gresh L, Mohamed-Hadley A, Ballesteros G, Davila MJ, Tellez Y, Sahoo MK, Balmaseda A, Harris E, Pinsky BA, 2016. Single-reaction multiplex reverse transcription PCR for detection of Zika, chikungunya, and dengue viruses. Emerg Infect Dis 22: 1295-1297.

17. Waggoner JJ et al., 2013. Comparison of the FDA-approved CDC DENV-1-4 real-time reverse transcription-PCR with a laboratorydeveloped assay for dengue virus detection and serotyping. J Clin Microbiol 51: 3418-3420. 